\title{
"NO SEA MALITO": UNA FÓRMULA DE CORTESÍA PARA ACTOS EXHORTATIVOS EN LA LITERATURA ECUATORIANA
}

ELKING ARAUJO*

Recibido: 29 de marzo de 2016 Aprobado: 6 de junio de 2016 



\section{"NO SEA MALITO": UNA FÓRMULA DE CORTESÍA PARA ACTOS EXHORTATIVOS EN LA LITERATURA ECUATORIANA}

Elking Araujo

Palabras clave: pragmática, cortesía verbal, literatura ecuatoriana, actos exhortativos.

Key words: pragmatics, verbal courtesy, Ecuadorian literature, hortatory acts.

\section{RESUMEN}

En toda lengua y cultura se construyen y usan fórmulas verbales que permiten mitigar los efectos impositivos provocados por los actos de habla. Estas fórmulas de cortesía son estudiadas por la pragmalingüística. La literatura —y en el caso particular de este artículo, la literatura ecuatoriana - suele recoger las fórmulas de cortesía propias del medio lingüístico al que pertenece el autor. Este artículo explora una de esas fórmulas en particular — la expresión "no ser malito"y sus variantes "no ser malo" $y$ "no ser asi" en un corpus de ochenta y un obras literarias de autores ecuatorianos del siglo XVIII al XXI, y analiza el tipo de estrategia cortés usada en cada fórmula, algunos elementos contextuales e implicaturas.

\section{ABSTRACT \\ Every language and culture} builds and uses verbal formulas in order to alleviate the effects imposed by the act of speaking. Pragmalinguistics studies these courtesy formulas. Literaturespecifically, Ecuadorian literature for this article-tends to use courtesy formulas that pertain to the author's linguistic field. This article explores one of these formulas in particular- the expression "no ser malito" and its variations " no ser malo" and " no ser asi" - in a corpus containing eighty-one literary works by Ecuadorian authors dating from the XVIII to the XXI century; and analyzes the courteous strategy used in each formula, some contextual elements and implicatures. 


\section{Aspectos teóricos generales de la cortesía verbal}

Aún criticada por su etnocentrismo, la teoría de la cortesía verbal de Brown y Levinson (1978 y su revisión en 1987) sigue constituyendo el referente más elaborado en los estudios de esta área de la pragmalingüística. En esta teoría, un concepto central es el de la imagen pública (en la lengua original de los autores, face). Esta consistiría en la autoimagen o prestigio que reclama para sí cada persona. Algunos críticos consideran que este concepto está determinado socioculturalmente y que, por tanto, su validez es relativa (Bravo, 2004, pág. 8). A pesar de esto, es la propuesta con mayor empaque teórico que permite explicar los actos de habla corteses. Siguiendo esta postura, la cortesía verbal consistiría en actos de habla dirigidos a mitigar la afectación de la imagen pública, tanto del hablante como del oyente. Pero Haverkate (1994) ha matizado que esos actos corteses no siempre responden a la necesidad de proteger la imagen pública de los hablantes: podrían responder a la justificación del acto de habla o a la valoración del coste verbal versus el beneficio interactivo (pág. 36).

En este sentido, la justificación del acto de habla está relacionada con las especificaciones que se dan para expresar la razón de haber realizado dicho acto. Y en cuanto al balance costo-beneficio, esto significa que un acto de habla se realiza con el menor costo verbal para conseguir el mayor beneficio u objetivo comunicativo.

Las diversas culturas han codificado expresiones y formas de cortesía verbal cuyos significados están vinculados siempre a la interpretación de elementos contextuales. Dicho de otro modo: las formas de cortesía de una comunidad hablante podrían pasar por descorteses en otra comunidad. $\mathrm{O}$ en ella misma, si los contextos así lo permiten.

La literatura como fuente de estudio de las formas de cortesía de una comunidad hablante tiene un valor importantísimo. Los autores son ineludiblemente hijos de una herencia lingüística de la comunidad a la que pertenecen y se convierten en replicadores y acrecentadores de dicha herencia. La lengua empleada en las obras literarias puede presentar un catálogo importante de formas y expresiones de cortesía verbal propias de la comunidad hablante en la que está enmarcada la producción literaria, y el estudio del contexto de uso de dichas formas permite establecer sus significados y el manejo que los hablantes hacen o han hecho de ellas.

\section{Una fórmula de cortesía en la literatura ecuatoriana: no sea malito.}

En un corpus de ochenta y un obras literarias de autores ecuatorianos 
(cuyas publicaciones van desde 1789 a 2015), la fórmula de cortesía para actos de habla exhortativos "no ser malito" o su femenino "no ser malita" tiene una recurrencia de veinte casos. Su registro más antiguo en esta selección de obras corresponde al año 1978 en que se publicaron dos obras que presentan por primera vez esta forma de cortesía (petición): la novela Bruna, Soroche y los tíos de Alicia Yánez Cossío (Quito, 1928) y el libro de cuentos Mala procesión de hormigas de Alejandro Carrión (Loja, 1915 Quito, 1992).

De acuerdo con la clasificación de las exhortaciones (entendidas como Actos amenazadores de imagen - AAlen actos impositivos y no impositivos), la expresión de marras sería un acto impositivo (el hablante quiere que el oyente realice el acto exhortado principalmente en beneficio del hablante mismo). Sea el caso de la siguiente muestra literaria:

vea, señor policía, de gana también pierde tiempo conmigo; qué saca; no me va a mandar a la cárcel ni tengo nada para darle; total, no tengo ni con qué pagar el permiso; no sea malito déjeme no más antes que me llamen (Valverde, 1986).

El hablante solicita al oyente (un policía) que lo deje en libertad: impone un acto al oyente en beneficio suyo mismo. Siguiendo a Haverkate, el hablante (con competencia social) debería emplear una estrategia de cortesía verbal que respete, "en lo posible, la imagen negativa de su interlocutor" (Haverkate, 1994, pág. 24). En este caso encontramos una estrategia verbal que pretende todo lo contrario: preservar la imagen positiva del oyente. La fórmula "no sea malito" conlleva una implicatura "sea bueno y haga lo que le pido". No realizar el acto solicitado por el hablante implicaría "ser malo": una consecuencia no deseada por el oyente pues afectaría su imagen positiva, es decir, como bien resume Escandell, "el deseo de ser apreciado por los demás, y de que otros compartan los mismos deseos" (Escandell Vidal, 1996, pág. 149).

Otro ejemplo del uso de esta fórmula de cortesía lo encontramos en la novela "Mientras llega el día" (2009) de Juan Valdano. En la historia presentada en esta novela, dos personajes, con cargos de funcionarios públicos, acuden donde un Conde, para realizar un acto exhortativo: le suplican que se haga cargo de la presidencia de la Real Audiencia. En un momento agitado de la vida pública del Ecuador — la novela, de corte histórico, está ambientada en la colonia prerrepublicana-, los mencionados funcionarios han participado en revueltas que concluyeron con la defenestra- 
ción del cargo del Conde. Pedirle que vuelva a asumir las responsabilidades de las que fue quitado encuentra dificultades en la condición de los hablantes: su pasado belicoso les desacredita moralmente, no tienen el poder suficiente para exigir que el oyente realice el acto de habla. La fórmula "no sea malito" busca, entonces, preservar, en primer término, la imagen positiva del Conde $y$, luego, revela la actitud sumisa y arrepentida de los hablantes:

Cuando el Manosalvas y el otro ministro, el Quintanilla, su compañero de aventuras, fueron a Inga-Quito, a la quinta del conde de Montejo, mi hermanito, para mala pata suya, también estuvo allí. Los dichos ministros fueron a rogarle al conde que no sea malito, que se haga no más cargo del gobierno de la Audiencia, que estaban muy arrepentidos, que el vivísimo del marqués de Villafranca había escapado dejándoles a ellos con ese carbón prendido entre las manos, que les perdone, que se olvide de todo, que ya ni más volverán a hacer revoluciones (Valdano, 2009).

La fórmula, al presuponer una condición censurable del oyente (ser malo), puede resultar altamente imposi- tiva. El sufijo apreciativo -ito aplicado al adjetivo malo tiene por función mitigar esta percepción.

Sin embargo, el corpus demuestra también el empleo de la fórmula sin el sufijo apreciativo -ito (no ser malo) que, a pesar de esto, aparece con menor frecuencia que "no sea malito". Aquí dos ejemplos. En la novela Los hijos de Alfonso Cuesta y Cuesta, un personaje solicita a otro un pedazo de tinaja para lo cual usa la estrategia de cortesía sin diminutivo "no ser malo":

-Oye -le decía en tanto el hijo de la hierbatera a Diegovos que eres tan bueno, puedes hasta decirme leproso... pero, no seas malo: dame un pedazo de la tinaja. No supo qué responderle (Cuesta y Cuesta, 1986).

En el libro "Catálogo de Ilusiones" de Raúl Serrano, un personaje masculino solicita a otro femenino que liquide una metafórica procesión de insectos para lo cual usa la fórmula de cortesía en género femenino sin diminutivo (no seas mala):

Las risas ya no son risas para el señor Octavio, son una procesión de hormigas gigantes, tarántulas que se le prenden en el cuello, me suplica, apresurado, que las liquide por amor a Dios, no seas 
mala, algún momento te lo pagaré, ¿me escuchas? (Serrano, 2006).

El criterio para preferir el uso entre "no ser malo" y "no ser malito" podría estar en uno de estos tres factores: el poder relativo, la distancia social o el grado de imposición. A mayor imposición del acto, o menor poder relativo del hablante sobre el oyente, o una mayor distancia social, se preferiría la forma con diminutivo. Compárense estos dos ejemplos que corresponden a las dos formas de cortesía ("no ser malo" frente a "no ser malito"):

Después vino la época en que me perseguía, se me pegaba, trataba de tocarme las nalgas, los senos, y me aguaitaba cuando me daba un baño en el cuarto de atrás, me decía que lo dejara entrar, no seas mala, un ratito, y yo me colocaba de espaldas para que no me viera la parte de adelante, diciéndole que iba a gritar, y entonces gritaba y él se iba (Donoso Pareja, 1982).

El personaje masculino, en este ejemplo de la novela de Donoso, es un muchacho que acosa a una de las empleadas domésticas de su casa, personaje femenino que es la voz narrativa. La condición de hijo del patrono le confiere poder sobre la oyente y su distancia social es mínima. La mujer lo ha tratado desde muy pequeño al punto de considerarlo su hijo. En virtud de la fórmula de cortesía escogida, el poder y la distancia social entre los dos personajes permite que se use la fórmula "no ser malo", sin diminutivo.

Por el contrario, en este otro ejemplo tomado de la novela "La frutilla mecánica", el padre de una jugadora colegial de fútbol ruega a una afamada jugadora profesional del mismo deporte que les acompañe en una celebración deportiva. La distancia social es grande: la jugadora profesional no ha tenido trato con el hablante (aunque este la conozca por su fama de seleccionada nacional). El poder relativo es nulo: el hablante no ostenta ninguna forma de poder sobre la oyente. De modo que el personaje masculino —el hablante en este ejemplo- debe usar la máxima forma de cortesía atenuada para ejecutar el acto impositivo: "no ser malito" con el sufijo apreciativo:

Pero no pudo negarse cuando fue mi padre en persona quien la llamó para pedirle que nos acompañara, no sea malita, mi Princesa no deja de hablar de usted, todos sabemos que es seleccionada nacional gracias a su trabajo en el equipo, además yo soy un admirador del Patogol Aguinaga, era del Quito pero era un gran 
jugador, por favor comparta con nosotros este festejo, es algo simple, un heladito nomás, ¿sí? (Ribadeneira, 2009).

La fórmula "no ser malito" (o, sin diminutivo, "no ser malo") presenta también una interesante variante: "no ser así". Esta nueva fórmula comparte la característica de estar dirigida también a preservar la imagen positiva del oyente. Está construida con una atenuación mayor que la fórmula "No ser malo", y no tendría relación su uso con la distancia social, el poder relativo o el grado de imposición del acto de habla. Obsérvese este ejemplo:

Ingeniero: vamos a dar una vuelta por el pueblo; ya ni me quiere responder, no sea así. Demos una vuelta inocente, y si me acepta, vamos a una tiendita o a un quiosco de adentro, de la parte buena, y nos tomamos una PiIsen, porque la cerveza es más rica sobre tierra firme (Díaz Icaza, 1978).

La estrategia de cortesía verbal que contiene esta fórmula, además de las características ya señaladas que comparte con "no ser malito" (consistir en una estrategia de cortesía positiva aun siendo un acto impositivo, implicar "ser malo" si no se realiza el acto en favor del hablante), radica en el uso del deíctico "así". Precisamente su carencia de significado atenúa el acto amenazador de imagen realizado por el hablante pero también la implicatura que presentaba su par "no ser malito", es decir, el oyente será, al no cumplir el mandato del hablante, algo indefinido pero que los elementos contextuales permiten interpretar como una condición o estado negativo, censurable socialmente. Y esto es así porque la exhortación está hecha para que el oyente, al realizarla, demuestre la condición esperada: como lo prefiere el hablante, es decir, colaborativo.

La María trató de separar al cojo y comenzó a tirarle del poncho. -jPor Dios! ¡Deja Juan! No seis así. Ya croque te has chumado. (Terán, 1986).

La fórmula de cortesía "no ser asi" para actos exhortativos puede relacionarse con el grado de distancia social con lo cual se prefiere el tuteo o no. A menor distancia social se prefiere el tú:

—Qué te pasa, Maruja, qué quieres, que la gente hable mal de mí. Si sin hacer nada también me calumnian como me calumnian, ahora, haciendo algo, dando motivo, ya ni para qué la vida, 
pues. - Ay, llámale, no seas así. Señor, señor, señor Correa, oiga, pst, pst, pst, señor Correa... (Dávila Vásquez, 2006).

En el ejemplo anterior, hablante y oyente se conocen y tienen trato íntimo. En virtud de aquello, Maruja (hablante) utiliza el pronombre tú en la forma "no ser así" para realizar el acto exhortativo "llama a Correa".

Si la distancia social es mayor de igual forma que en el resto de fórmulas de tratamiento del español del Ecuador - se prefiere la forma dirigida al pronombre "usted".

Cuando él le sonrió, no pudo impedirse sonreírle también. Llegaban a la bocacalle de su casa y seguía tras ella. Entonces se volvió, pidiéndole: -¡No sea así, váyase ya, que mi mamá va a verlo! (Gallegos Lara, 2003).

En este último ejemplo, el personaje femenino marca la distancia con el sujeto que la pretende a través del uso consistente del "usted" en los verbos "váyase" y "va", uso que se extiende por supuesto a la fórmula de cortesía "no sea así".

\section{Conclusiones}

El análisis de la fórmula de cortesía "No ser malito" y sus variantes "no ser malo"y "no ser así" revelan aspectos pragmáticos contrarios a los propuestos por Haberkate en el sentido de que se caracterizan por ser estrategias de cortesía positiva. Añaden a su estructura aspectos particulares del habla ecuatoriana: la mitigación de los actos impositivos a través del uso del sufijo apreciativo -ito/ita y la alternación del tuteo o del uso del pronombre "usted". 


\section{Bibliografía}

Bravo, D. (2004). Panorámica breve acerca del marco teórico y metodológico. En D. Bravo, \& A. Briz (Edits.), Pragmática sociocultural: estudios sobre el discurso de cortesía en español (pág. 397). Barcelona: Madrid.

Cuesta y Cuesta, A. (1986). Los hijos. Quito: Oveja Negra.

Dávila Vásquez, J. (2006). La noche maravillosa. Quito: Libresa.

Díaz Icaza, R. (1978). Por la tierra. Quito: Casa de la Cultura Ecuatoriana.

Donoso Pareja, M. (1982). Nunca más el mar. Quito: El Conejo.

Escandell Vidal, M. V. (1996). Introducción a la pragmática. Barcelona: Ariel. Gallegos Lara, J. (2003). Las cruces sobre el agua. Quito: Casa de la Cultura Ecuatoriana.

Haverkate, H. (1994). La cortesía verbal. Madrid: Gredos.

Ribadeneira, A. (2009). La frutilla mecánica. Quito: Eskeletra.

Serrano, R. (2006). Catálogo de llusiones. Quito: Eskeletra.

Terán, E. (1986). El cojo Navarrete. Bogotá: Oveja Negra.

Valdano, J. (2009). Mientras llega el día. Quito: Campaña Nacional Eugenio Espejo por el Libro y la Lectura.
Valverde, A. B. (1986). Herederás un mar que no conoces y lenguas que no sabes. Quito: El Conejo. 